\title{
Peritonitis tuberculosa con perforación intestinal en población pediátrica en un hospital de tercer nivel de Bogotá, Colombia
}

\author{
Germán Mogollón ${ }^{1}$, Pablo Luengas $^{1}$, Carlos Beltrán ${ }^{1}$, Marcos Rottermann $^{1}$, Sergio Del Río ${ }^{1}$, \\ SANDRA Bresciani ${ }^{2}$, Luis Felipe CABrera ${ }^{3}$
}

Palabras clave: Mycobacterium tuberculosis; tuberculosis gastrointestinal; peritonitis, peritonitis tuberculosa; intestino delgado; perforación intestinal.

\section{Resumen}

Introducción. La peritonitis tuberculosa es un tipo de presentación poco frecuente de la tuberculosis en población pediátrica, con enfermedades concomitantes que favorecen una inmunosupresión crónica. En el presente articulo, se presenta un caso de peritonitis tuberculosa manejado en nuestra institución y se hace una revisión crítica de la literatura científica.

Materiales y métodos. Se llevó a cabo un estudio observacional y descriptivo de los pacientes que fueron sometidos a cirugía con hallazgo intraoperatorio de

1 Cirugía pediátrica, Hospital Simón Bolívar, Bogotá, D.C., Colombia

2 Cuidado Intensivo Pediátrico, Hospital Simón Bolívar, Bogotá, D.C., Colombia

3 Residente de Cirugía General, Universidad El Bosque, Bogotá, D.C., Colombia

Fecha de recibido: 11 de julio de 2016

Fecha de aprobación: 7 de septiembre de 2016

Citar como: Mogollón G, Luengas P, Beltrán C, Rottermann, Del Río S, Bresciani S, et al. Peritonitis tuberculosa con perforación intestinal en población pediátrica en un hospital de tercer nivel de Bogotá, Colombia. Rev Colomb Cir. 2017;32:94-101. peritonitis generalizada, y con diagnóstico confirmado de peritonitis tuberculosa, desde el $1^{\circ}$ de junio del 2004 hasta el 30 de junio del 2015.

Resultados. Se revisaron 486 pacientes con peritonitis generalizada, de los cuales solo a un paciente masculino de cuatro años, con síndrome nefrótico y tuberculosis pulmonar, se le hizo diagnóstico de peritonitis tuberculosa, con perforación intestinal y necesidad de cirugía de urgencia. Falleció el día 51 de hospitalización en la unidad de cuidados intensivos.

Discusión y conclusiones. La tuberculosis peritoneal es una entidad poco frecuente en la población pediátrica, pero debe considerarse en pacientes con factores de riesgo para inmunosupresión, nexo de contagio para tuberculosis y ascitis, acompañados de dolor abdominal y fiebre. El diagnóstico temprano mejora el pronóstico del paciente y la biopsia peritoneal es la prueba estándar para confirmar esta entidad. Su incidencia en nuestra institución es de 0,2\%.

\section{Introducción}

Aún hoy en día, la tuberculosis es una causa importante de morbimortalidad a nivel mundial. La peritonitis tuberculosa es una presentación poco frecuente de la tuberculosis en la población pediátrica y predomina en los pacientes con enfermedades concomitantes, como cirrosis, diabetes mellitus de tipo 1, falla renal crónica 
con diálisis peritoneal que permite una inoculación directa abdominal, desnutrición, enfermedades autoinmunitarias y HIV, que generan estados de inmunosupresión crónica y favorecen la diseminación hematógena con colonización de los ganglios linfáticos mesentéricos. ${ }^{1,2}$

La mayoría de los casos se presentan en la tercera y cuarta décadas de la vida, con una incidencia de 0,1 a $3,5 \%$ de todos los pacientes, con un pico de edad entre los 35 y 45 años $^{3}$. De este porcentaje, Forssbohm, et al., reportaron solo un $5 \%$ en población por debajo de los 14 años en Alemania, lo cual representa de 4 a $10 \%$ de todas las presentaciones extrapulmonares; se inician con un cuadro clínico inusual de dolor abdominal crónico y difuso, lo que no facilita el diagnóstico, el cual se hace de forma tardía, lo cual aumenta las complicaciones y la mortalidad en estos pacientes ${ }^{4}$.

En el presente artículo, se presenta un caso de peritonitis tuberculosa manejado en nuestra institución y se hace una revisión crítica de la literatura científica.

\section{Materiales y métodos}

Se llevó a cabo un estudio observacional, descriptivo y retrospectivo, en un hospital de tercer nivel, de los pacientes con edades entre los 1 y 14 años que fueron sometidos a cirugía por abdomen agudo en el Servicio de Cirugía Pediátrica, con el hallazgo intraoperatorio de peritonitis generalizada y diagnóstico final de peritonitis tuberculosa, del $1^{\circ}$ de junio del 2004 al 30 de junio del 2015.

Se seleccionó este caso para presentarlo y se revisó la historia clínica.

\section{Resultados}

Se encontraron 486 pacientes intervenidos por abdomen agudo y peritonitis generalizada, entre los cuales solo hubo un niño de cuatro años de edad, proveniente de un área rural endémica para tuberculosis. Tenía antecedentes de síndrome nefrótico y consumo crónico de corticoesteroides, con exposición familiar a la tuberculosis, pobreza extrema y diagnóstico de tuberculosis pulmonar no tratada (figura 1).

Presentó un cuadro clínico de dolor abdominal, obstrucción intestinal y ascitis de un mes evolución; además, síndrome de respuesta inflamatoria sistémica que produjo taquicardia y fiebre. Se requirió su hospitalización y la práctica de una laparotomía de urgencia. En la ecografía abdominal total se encontró líquido libre en la cavidad abdominal y, la tomografía computadorizada de abdomen contrastada con medio hidrosoluble, un neumoperitoneo masivo (figura 2).

La prueba ELISA fue negativa para HIV, la adenosina deaminasa (ADA) en líquido peritoneal fue mayor de 40 U/L y la coloración de Ziehl-Neelsen fue positiva para Mycobacterium tuberculosis (figura 3). En la histopatología se reportaron granulomas de necrosis de caseificación en la biopsia de ganglio mesentérico ${ }^{5}$, úlceras en la pared intestinal y microtrombos en los vasos sanguíneos del mesenterio.

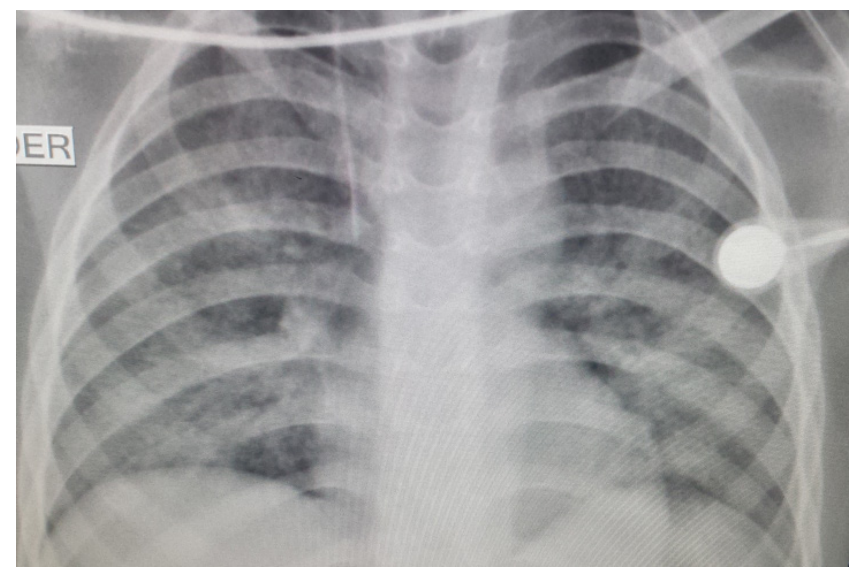

FIGURA 1. Tuberculosis pulmonar: radiografía de tórax con opacidades en vidrio esmerilado en ambos capos pulmonares

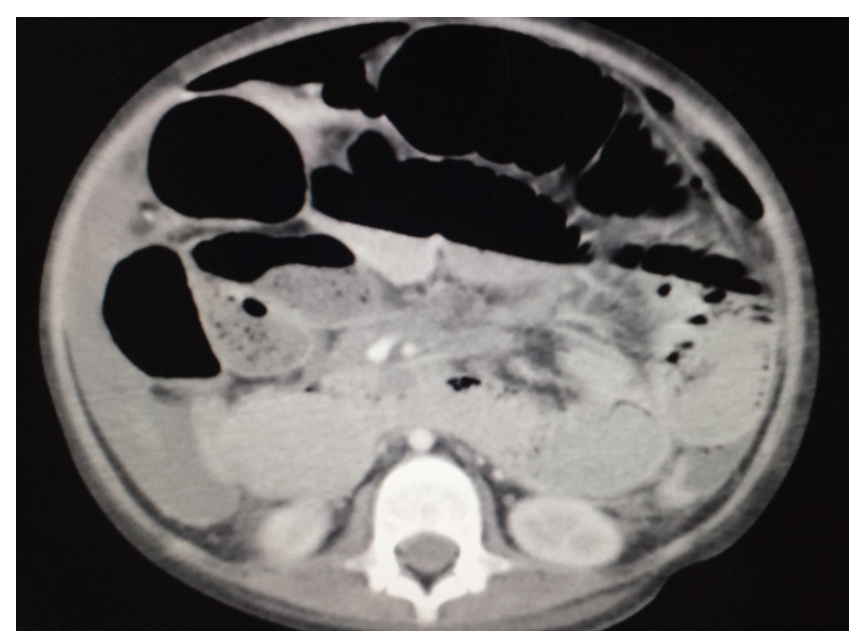

Figura 2. Neumoperitoneo: TC de abdomen con aire interasas y signos de obstrucción intestinal dados por niveles hidroaéreos y distensión de asas delgadas. 


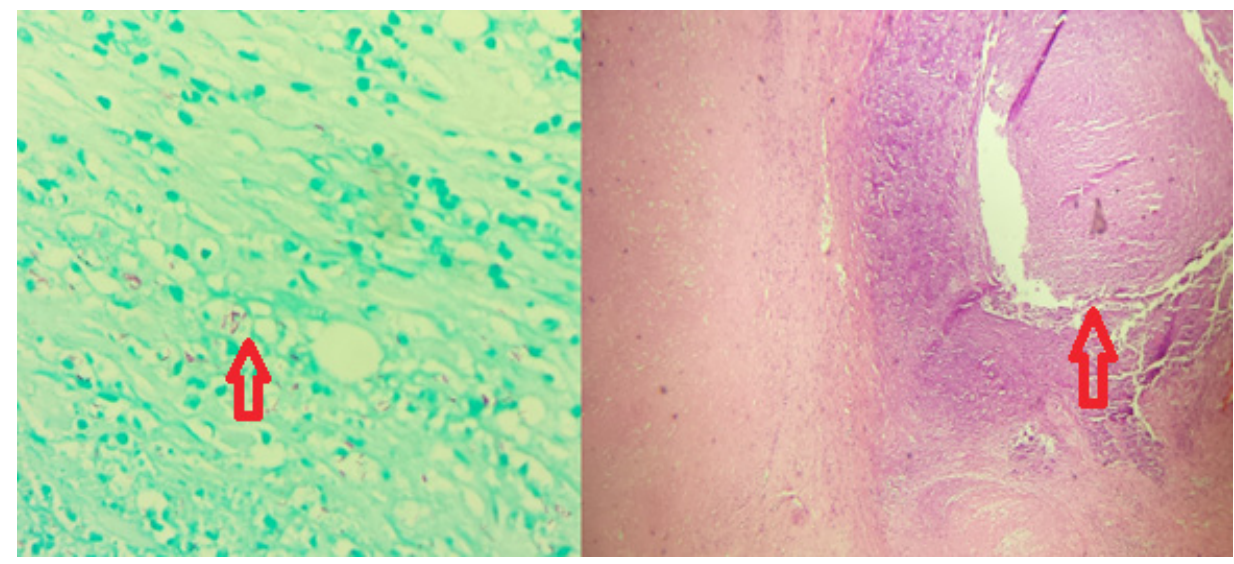

Figura 3. A. Bacilos ácido-alcohol resistentes. Coloración Ziehl-Neelsen a 100X B. Granulomas con necrosis de caseificación. Hematoxilina-eosina $100 \mathrm{X}$

Durante la cirugía, se observó gran distensión de las asas intestinales delgadas, importante proceso de adherencias interasas, múltiples perforaciones a lo largo de todo el yeyuno, el íleon y el ciego, peritonitis fecal y múltiples ganglios con necrosis de caseificación en el mesenterio (figura 4). Se practicó una cirugía de control de daños para la sepsis de origen abdominal, con drenaje de la peritonitis fecal, resección de yeyuno distal, íleon y ciego, cierre temporal del colon ascendente, tres enterorrafias y yeyunostomía derivativa. Se requirieron 15 lavados quirúrgicos. Las fístulas intestinales originadas en las enterorrafias del yeyuno se trataron con sellador de fibrina, y se administró nutrición parenteral total y octreótido.

A la sexta semana posoperatoria, presentó estenosis intestinal en dos de las tres enterorrafias, las cuales se resolvieron quirúrgicamente. El tratamiento médico incluyó corticoesteroides y antituberculosos intravenosos, con carbapenémicos y fluoroquinolonas. El paciente desarrolló una púrpura fulminante (figura 5). La hospitalización se prolongó por 51 días, hasta la muerte del niño, con una incidencia de $0,2 \%$ de peritonitis tuberculosa en este estudio.

\section{Discusión}

En los Estados Unidos, la tuberculosis peritoneal corresponde solo al $0,3 \%$ de todos los casos de tuberculosis en niños, similar a la evidenciada en el presente estudio $(0,2 \%)$, con una edad media de 13 años.

La edad del paciente de este informe, cuatro años, no concuerda con el promedio de las series reportadas

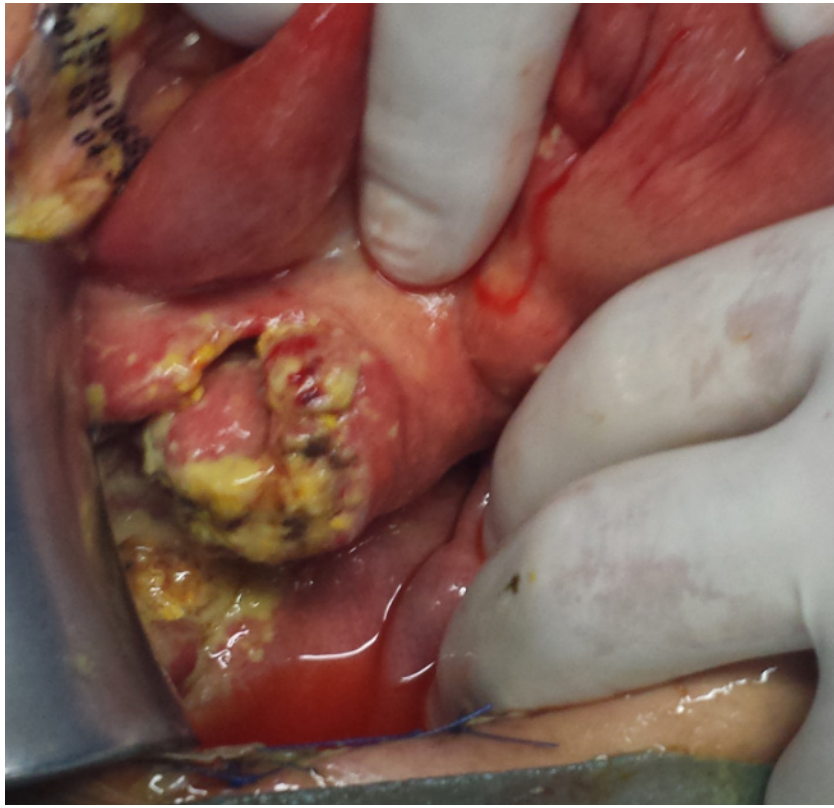

Figura 4. Ganglios con necrosis de caseificación en el mesenterio

en la literatura científica, la cual varía de 12 a 16 años, con una media de 14,2. Solamente Tanrikulu, et al., en el 2005 en Turquía, habían reportado pacientes con edades similares en los últimos 15 años ${ }^{5}$.

En Colombia, anualmente se reportan, aproximadamente, 24 a 26 casos de tuberculosis por cada 100.000 habitantes, con una mortalidad de 2,1 por cada 100.000 habitantes estimada en el 2010, con una incidencia de $6 \%$ en la población menor de 15 años; la tuberculosis extrapulmonar más frecuente es la pleural seguida de la ganglionar y no existen reportes de tuberculosis peritoneal 

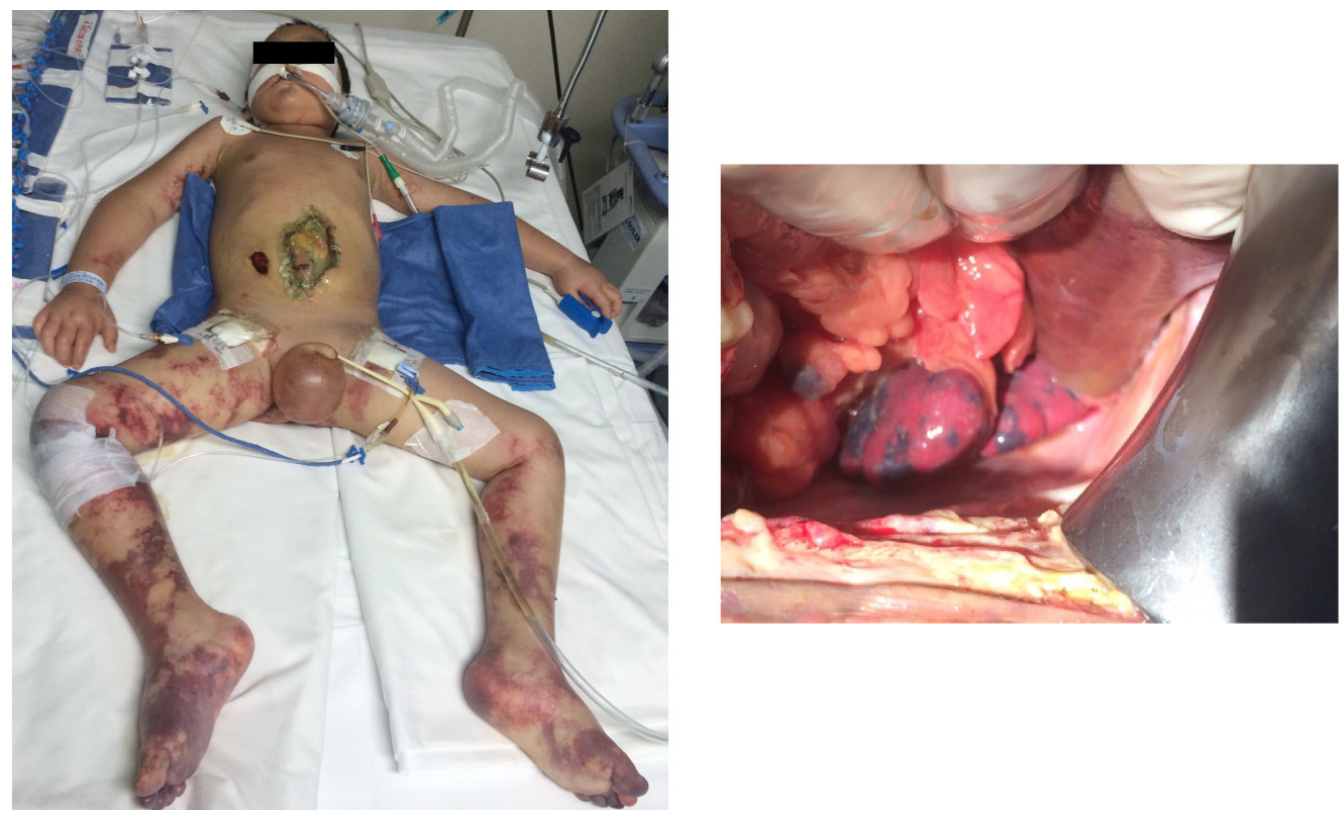

Figura 5. Púrpura fulminante en extremidades y bazo

en niños. Dulger, et al., reportaron una mortalidad de 10 a $25 \%$ en adultos; no se encontró reporte del porcentaje de mortalidad en población pediátrica ${ }^{6}$.

Definitivamente, debido a la epidemia mundial de HIV, el amplio uso de esteroides, las enfermedades renales y las hepáticas crónicas, la tuberculosis peritoneal ya no es una enfermedad del tercer mundo, y su incidencia y prevalencia en nuestro país no varía mucho con respecto a los países desarrollados ${ }^{3,4}$.

Los bacilos pueden colonizar el peritoneo a través del tubo digestivo, invadiendo los ganglios linfáticos mesentéricos, como lo reportaron Dulger, et al., en una serie de 21 pacientes, por ingestión de leche no pasteurizada o de esputo contaminados con Mycobacterium bovis, por vía sanguínea o por inoculación directa durante la diálisis peritoneal ${ }^{6}$.

Existen tres formas de presentación clínica: húmeda, seca y fibrótica. La forma más frecuente de la enfermedad es la peritonitis húmeda, con formación de múltiples ganglios tuberculosos en el peritoneo visceral y parietal, con aumento en la producción de líquido peritoneal que genera ascitis, como se observó en nuestro caso ${ }^{6-8}$.

Los síntomas de la tuberculosis peritoneal son inespecíficos, se presentan con una evolución de semanas e incluso meses, y consisten en distensión abdominal secundaria a ascitis $(70 \%)$, dolor abdominal $(55,5 \%)$, defensa abdominal $(48 \%)$, fiebre $(44,4 \%)$ y pérdida de peso (33,3 a $61 \%)$. En el presente caso, se presentó obstrucción intestinal debido a un avanzado proceso de adherencias secundario a la inflamación crónica generada por la peritonitis tuberculosa, la cual se reporta en otras series en la literatura, como las de Ozbey y Akcakaya ${ }^{9,10}$.

Esto hace que el diagnóstico se haga tardíamente, con un promedio de 2,6 meses entre el inicio de los síntomas y el diagnóstico; en este caso, fue inferior a lo reportado en la literatura científica. Es importante establecer si ha existido contacto con individuos con tuberculosis activa, ya que hasta $66,7 \%$ de los pacientes con tuberculosis peritoneal han estado expuestos, como en este caso. Además, la peritonitis tuberculosa debe incluirse en el diagnóstico diferencial en casos de ascitis de origen no claro, como lo recomiendan Sanai, et al., en una revisión sistemática ${ }^{3}$. La prueba de tuberculina es positiva en 18 a $27 \%$ de los pacientes, como lo informaron Muneef, et $a l^{11}$. Los hallazgos físicos y radiológicos de tuberculosis están presentes en 50 a $75 \%$ de los casos, como informaron Cruz, et al., y se asocian con derrame pleural en 12 a $63 \%$, como en el presente caso ${ }^{12,13}$.

En la mayoría de las series no se observaron bacilos ácido-alcohol resistentes en el líquido peritoneal; en este 
paciente, las baciloscopias del líquido peritoneal fueron positivas. En el análisis de líquido peritoneal mediante paracentesis, se observó un exudado con predominio de linfocitos, el gradiente de albúmina fue menor de $1,1 \mathrm{~g} / \mathrm{dl}$, la adenosina deaminasa (ADA) fue positiva, por encima de 40 UI/L (sensibilidad de $100 \%$ y especificidad de 97 $\%$ ), y la PCR para M. tuberculosis fue positiva; su utilidad no está bien establecida, pero en una serie de 11 casos fue positiva en todos. El antígeno CA-125, marcador tumoral, es un indicador fuerte de tuberculosis peritoneal cuando hay una neoplasia maligna asociada; se aumenta en casos de serositis, cuando se afecta la pleura o el peritoneo. La elevación del CA-125 obliga a descartar otras enfermedades asociadas al cuadro clínico ${ }^{14-16}$.

Para el diagnóstico imaginológico, se puede emplear la ecografía abdominal total, la cual permite detectar líquido libre con tabiques finos y linfadenopatías con centro hipoecogénico, que corresponde a necrosis de caseificación. En la tomografía computadorizada de abdomen se aprecia engrosamiento de las asas, especialmente el íleon y la válvula ileocecal, con nódulos en el mesenterio, líquido libre, signos de obstrucción intestinal y perforación ${ }^{17-19}$.

En la ecografía de este paciente se encontró líquido libre, escaso pero sin tabiques y no se evidenciaron adenomegalias, mientras que en la tomografía se observaron signos de obstrucción intestinal con perforación ${ }^{20,21}$. La colonoscopia se puede emplear con el fin de detectar lesiones en la válvula ileocecal y en el íleon distal; sin embargo, los hallazgos pueden ser similares a los de la enfermedad de Crohn, como lo reportan $\mathrm{Wu}$, et al. En la esofagogastroduodenoscopia no se demostraron ulceraciones sugestivas de tuberculosis ${ }^{22-24}$.

Las complicaciones incluyen obstrucción intestinal (31,7\%), perforación intestinal (4,9\%), fístula enterocutánea $(2,4 \%)$ y vólvulo intestinal secundario a la linfadenitis mesentérica $(2,4 \%)$, como las descritas en este caso.

En 1964, Paustian, et al., establecieron los criterios para el diagnóstico de tuberculosis abdominal que siguen siendo útiles ${ }^{14}$ :

1) evidencia histológica de tuberculomas y necrosis caseosa;
2) evidencia histológica en los ganglios mesentéricos extirpados;

3) crecimiento de $M$. tuberculosis en el cultivo, y

4) demostración de bacilos ácido-alcohol resistentes en la biopsia peritoneal, la cual se debe tomar por vía laparoscópica cuando no hay una indicación quirúrgica de urgencia.

Las indicaciones para cirugía de urgencia incluyen irritación peritoneal, deterioro clínico con signos de respuesta inflamatoria, hipotensión y obstrucción intestinal o hallazgos radiológicos de perforación intestinal. En la cirugía se puede encontrar engrosamiento del peritoneo, con conglomerados de linfadenopatías y adherencias moderadas o graves. Se recomienda practicar una biopsia por escisión de un ganglio peritoneal, la cual permite confirmar el diagnóstico al demostrar granulomas de caseificación y bacilos ácido-alcohol resistentes. En $15 \%$ de los adultos se produce perforación intestinal. En países endémicos como la India, 3,9 a $10 \%$ de todas las perforaciones del intestino delgado son secundarias a tuberculosis intestinal y peritoneal ${ }^{13}$.

Existen tres tipos histopatológicos de tuberculosis intestinal: ulcerosa, hipertrófica y úlcero-hipertrófica. La ulcerosa afecta comúnmente el íleon y el yeyuno, y se caracteriza por una o varias úlceras transversales; su cicatrización conduce a la estenosis, o pueden perforarse, sangrar y formar fístulas. La tuberculosis hipertrófica y la úlcero-hipertrófica comúnmente afectan la región ileocecal, causando obstrucción o formando una masa. La mayoría de las perforaciones se deben a la obstrucción distal secundaria a estenosis mayores de $12 \mathrm{~cm}$ o múltiples, como lo reportaron Sharma, et $a l .{ }^{22}$, y no a una perforación directa de la úlcera. La predilección del bacilo por la región ileocecal se atribuye a tres factores, principalmente: la estasis fisiológica de la zona, la alta tasa de absorción, que permite el contacto libre del microorganismo con la mucosa, y la abundancia de tejido linfoide en este sitio ${ }^{14,15}$.

Es importante saber que la ingestión crónica de corticosteroides se asocia con un mayor riesgo de perforación intestinal e infección, reactivación y difusión de la tuberculosis. En un estudio retrospectivo de casos y controles de 12 pacientes, Ara, et al. ${ }^{21}$, practicaron siete suturas primarias; se presentaron tres casos de 
dehiscencia parcial de la sutura y cinco resecciones y anastomosis término-terminal, sin ninguna dehiscencia. El sitio de perforación más frecuente fue el íleon y solo cuatro paciantes presentaron múltiples perforaciones. $\mathrm{La}$ mortalidad global fue de $25 \%{ }^{16,17}$.

En nuestro caso se requirió una resección intestinal amplia por la gran cantidad de perforaciones. Debido al gran riesgo de un síndrome de intestino corto y la necesidad de un trasplante intestinal en las reintervenciones posteriores, se decidió preservar la mayor longitud posible de intestino delgado y grueso, haciendo una sutura primaria. Sin embargo, al tratarse de una tuberculosis intestinal ulcerosa, se favoreció la formación de estenosis de las enterorrafias y se requirieron enteroplastias para resolverlas. Se hicieron revisiones quirúrgicas periódicas por el alto riesgo de fístulas intestinales, las cuales, posiblemente, son causadas por microtrombos en el mesenterio que generan hipoperfusión intestinal, isquemia y necrosis en las suturas, y favorecen su formación ${ }^{18,19}$.

Con respecto a la fisiopatología de la perforación, es importante saber que se asocia más frecuentemente con el SIDA que, al disminuir la inmunidad, disminuye también la fibrosis y, por lo tanto, se reduce la cicatrización. Se ha observado una mayor incidencia de perforación en los pacientes inmunocomprometidos que reciben tratamiento antituberculoso, debido a la reducción de la respuesta inflamatoria por los medicamentos, antes de que ocurra la fibrosis adecuada, lo que lleva a una mala cicatrización de las úlceras o a una reacción paradójica inducida por fármacos, causada por alteraciones locales de la inmunidad en relación con los productos metabólicos de las micobacterias ${ }^{20,21}$.

La mayoría de los pacientes son de un estrato socioeconómico bajo, lo cual hace pensar que es posible que la desnutrición, y la consecuente disminución de la inmunidad mediada por células, de la fibrosis y de la cicatrización, sean un factor en la patogénesis y el aumento de la frecuencia de perforación intestinal, como lo reportan Dasgupta, et al. ${ }^{25}$. En $40 \%$ de los pacientes se producen perforaciones múltiples, lo cual se asocia con mal pronóstico y mortalidad por encima del $30 \%$, posiblemente secundaria a sepsis de origen abdominal, peritonitis terciaria, resección intestinal masiva e imposibilidad de iniciar el tratamiento oral antituberculoso estándar con rifampicina, isoniacida, pirazinamida y etambutol ${ }^{22,23}$.
La vasculitis es una característica bien documentada de la tuberculosis del sistema nervioso central, la pulmonar y la renal. Sin embargo, en pocos estudios se ha evaluado el papel de la vasculitis mesentérica en la historia natural de la perforación intestinal, la cual puede conducir a la isquemia intestinal, lo que contribuye al desarrollo de úlceras, fibrosis, estenosis y, por último, perforación. Se han observado cambios en los vasos mesentéricos grandes, medianos y pequeños, en pacientes con diagnóstico histológico de tuberculosis intestinal que presentan obstrucción y perforación; incluyen granulomas adyacentes a la pared de los vasos, endarteritis submucosa, formación de trombos en los vasos grandes y medianos, y fibrosis de la subíntima. Cabe resaltar que no hay reportes de casos recientes de perforación intestinal en niños secundaria a tuberculosis peritoneal ${ }^{24}$.

El tratamiento médico se basa en la administración de antituberculosos orales (rifampicina, isoniacida, pirazinamida y etambutol), si el paciente los tolera, o la de antituberculosos intravenosos (carbapenémicos, aminoglucósidos y fluoroquinolonas), asociada a la de corticoesteroides intravenosos; este manejo debe iniciarse tan pronto exista la sospecha clínica de peritonitis tuberculosa, ya que puede disminuir el porcentaje de complicaciones y la estancia hospitalaria ${ }^{25,26}$. Sin embargo, el presente paciente presentó fístulas intestinales posoperatorias y estenosis intestinal, las cuales requirieron nutrición parenteral total, octreótido, sutura y enteroplastia con sellador de fibrina.

\section{Conclusiones}

La tuberculosis peritoneal es poco frecuente en la población pediátrica, pero debe sospecharse en pacientes con factores de riesgo para inmunosupresión, noción de contagio para tuberculosis y ascitis, acompañados de dolor abdominal y fiebre. La cirugía está indicada en pacientes sin evidencia de perforación o estenosis, para la toma de biopsia peritoneal con el fin de obtener confirmación histológica y debe hacerse, preferiblemente, por vía laparoscópica si los hallazgos lo permiten. En caso de perforación intestinal, se debe practicar resección intestinal y anastomosis término-terminal, si no existe riesgo de síndrome de intestino corto y necesidad de trasplante intestinal, con el fin de disminuir la posibilidad de fístulas y estenosis. 
El inicio temprano del tratamiento oral estándar antituberculoso con rifampicina, isoniacida, pirazinamida y etambutol en el posoperatorio es ideal para mejorar la evolución clínica del paciente. Esta enfermedad exige un equipo multidisciplinario con gran compromiso clínico y con múltiples opciones terapéuticas abiertas a la innovación. Sin embargo, se requieren más estudios para definir el manejo ideal de la perforación intestinal en esta enfermedad y establecer guías de tratamiento estandarizadas en la población pediátrica.

\title{
Tuberculous peritonitis with intestinal perforation in the pediatric population at a third level of care hospital in Bogotá, Colombia.
}

\begin{abstract}
Introduction: Tuberculous peritonitis is a rare type of presentation of tuberculosis in pediatric patients with comorbidities associated with chronic immunosuppression. In this article, we present a case of tuberculous peritonitis managed at our institution and a critical review of the literature.

Materials and methods: Descriptive observational study of patients who underwent surgery with the intraoperative finding of generalized peritonitis in the period June 1, 2004 to June 30, 2015. Only one patient was confirmed with tuberculous peritonitis.

Results: 486 patients with generalized peritonitis were studied. The diagnosis of tuberculous peritonitis was confirmed only in a 4 year old male patient with nephrotic syndrome and pulmonary tuberculosis. The patient had intestinal perforation and required an emergency surgical intervention. He died on day 51 of hospitalization in the ICU.

Discussion and conclusions: Peritoneal tuberculosis is a rare entity in the pediatric population, but it should be considered in patients with risk factors for immunosuppression, nexus of tuberculosis infection and ascites, accompanied by abdominal pain and fever. Early diagnosis improves the patient's prognosis, and peritoneal biopsy is the gold standard to confirm this entity, whose incidence is $0.2 \%$ at our institution.
\end{abstract}

Key words: Mycobacterium tuberculosis; tuberculosis, gastrointestinal; peritonitis, tuberculous; intestine, small; intestinal perforation.

\section{Referencias}

1. Dinler G, Sensoy G, Helek D, Kalayci AG. Tuberculous peritonitis in children: Report of nine patients and review of the literature. World J Gastroenterol. 2008;14:7235-9.

2. Niu W, Li M, Wan Y,Wang H, Wu Z, Cui J. A case of tuberculous peritonitis. Exp Ther Med. 2012;4:1104-6.

3. Sanai F, Bzeizi K. Systematic review: tuberculous peritonitis-presenting features, diagnostic strategies and treatment. Aliment Pharmacol Ther. 2005;22:685-700.

4. Forssbohm M, Zwahlen M, Loddenkemper R, Rieder HL. Demographic characteristics of patients with extrapulmonary tuberculosis in Germany. Eur Respir J. 2008;31:99-105.

5. Tanrikulu AC, Aldemir M, Gurkan F, Suner A, Dagli CE, Ece A. Clinical review of tuberculous peritonitis in 39 patients in Diyarbakir, Turkey. J Gastroenterol Hepatol. 2005;20:906-9.

6. Dulger AC, Karadas S, Mete R, Türkdogan MK, Demirkiran D, Gültepe B. Analysis of cases with tuberculous peritonitis: A single-center experience. Turk J Gastroenterol. 2014;25:72-8.
7. Instituto Nacional de Salud, Colombia. Fecha de consulta: 30 de julio de 2015. Disponible en: www.ins.gov.co/noticias/ paginas/¡vivamos-sin-tuberculosis!.aspx\#.VbpwPl_Okp.

8. Patel SM, Sweetser S. The wet-ascitic form of tuberculous peritonitis. Hepatology. 2011;54:364-5.

9. Ozbey H, Tireli GA, Salman T. Abdominal tuberculosis in children. Eur J Pediatr Surg. 2003;13:116-9.

10. Akcakaya A, Sahin M, Coskun A, Demiray S. Comparison of mechanical bowel obstruction cases of intra-abdominal tumor and non-tumoral origin. World J Surg. 2006;30:1295-9.

11. Muneef MA, Memish Z, Mahmoud SA, Sadoon SA, Bannatyne $\mathrm{R}$, Khan Y. Tuberculosis in the belly: A review of forty-six cases involving the gastrointestinal tract and peritoneum. Scand J Gastroenterol. 2001;36:528-32.

12. Uzunkoy A, Harma M, Harma M. Diagnosis of abdominal tuberculosis: Experience from 11 cases and review of the literature. World J Gastroenterol. 2004;10: 3647-9. 
13. Cruz AT, Starke JR. Clinical manifestations of tuberculosis in children. Paediatr Respir Rev. 2007;8:107-17.

14. Paustian FF. Tuberculosis of the intestine. En: Bockus HL, editor. Gastroenterology, 2nd edition. Philadelphia: WB Saunders; 1964. p. 311-34.

15. Sotoudehmanesh R, Shirazian N, Asgari AA, Malekzadeh R. Tuberculous peritonitis in an endemic area. Dig Liver Dis. 2003;35:37-40.

16. Kocaman O. Understanding tuberculous peritonitis: A difficult task to overcome. Turk J Gastroenterol. 2014;25:79-80.

17. Kocaman O, Danalioğlu A, İnce AT, Tozlu M, Şentürk H. Diagnosis of tuberculous peritonitis using endoscopic ultrasoundguided fine-needle aspiration biopsy of the peritoneum. Turk J Gastroenterol. 2013;24:65-9.

18. Uygur-Bayramicli O, Dabak G, Dabak R. A clinical dilemma: Abdominal tuberculosis. World J Gastroenterol. 2003;9:1098101.

19. Bhargava DK, Shriniwas, Chopra P, Nijhawan S, Dasarathy S, Kushwaha AK. Peritoneal tuberculosis: laparoscopic patterns and its diagnostic accuracy. Am J Gastroenterol. 1992;87:10912.

20. Rasheed S, Zinicola R, Watson D, Bajwa A, McDonald PJ. Intra-abdominal and gastrointestinal tuberculosis. Colorectal Dis. 2007;9:773-783.

21. Ara C, Sogutlu G, Yildiz R, Kocak O, Isik B, Yilmaz S, et al. Spontaneus small bowel perforations due to intestinal tubercu- losis should not be repaired by simple closure. J Gastrointest Surg. 2005;9:514-7.

22. Sharma MP, Bhatia V. Abdominal tuberculosis. Indian J Med Res. 2004;120:305-15.

23. Šefr R, Rotterová P, Konecný J. Perforation peritonitis in primary intestinal tuberculosis. Dig Surg. 2001;18:475-9.

24. Wu YF, Ho CM, Yuan CT, Chen CN. Intestinal tuberculosis previously mistreated as Crohn's disease and complicated with perforation: A case report and literatura review. Springerplus. 2015;4:326.

25. Dasgupta A, Singh N, Bhatia A. Abdominal Tuberculosis: A histopathological study with special reference to intestinal perforation and mesenteric vasculopathy. J Lab Physicians. 2009;1:56-61.

26. Coccolini F, Ansaloni L, Catena F, Lazzareschi D, Puviani L, Pinna AD. Tubercular bowel perforation: what to do? Ulus Travma Acil Cerrahi Derg. 2011;17:66-74.

27. Borrajo M, Pérez C, Novoa EF, Iglesias A, Camba A, Bravo JJ, et al. Peritonitis tuberculosa en diálisis peritoneal. Nefrología 2009;29:170-2.

Correspondencia: Luis Felipe Cabrera, MD

Correo electrónico: luis.felipe.cabrera@hotmail.com Bogotá, D.C., Colombia 